\title{
Transition from the Chaotic Turbulence to the Turbulent Structures
}

\author{
A. KINGSEP \\ Russian Research Center 'Kurchatov Institute' 123182, Moscow, Russia
}

(Received 1 March 1999)

\begin{abstract}
Weak turbulence, similar to incoherent light, may be represented as an ensemble of quasifree quanta or Fourier harmonics. Unlike it, strongly turbulent state should be based on nonlinear structures. In particular, strong plasma turbulence may be constructed of discrete formations, viz., Langmuir solitons. Instead for 'infrared catastrophe' typical of the weakly turbulent regime, one deals with the 'relay race' model providing the proper direction of the energetic flux over scales from the source towards the leakage.
\end{abstract}

Keywords: Strong turbulence, Nonlinear structures, Langmuir soliton, 'relay race' scenario

\section{INTRODUCTION}

This paper is devoted to the problem of the nonlinear structures as manifestations of the strongly turbulent behavior. Transition from the weak (chaotic, perturbational) turbulence to the nonlinear structures is demonstrated on the basis of the strong Langmuir turbulence. First such a model had been proposed in [1], further results were presented in $[2,3]$.

In many problems of plasma physics, solid state physics, hydrodynamics of the surface waves etc., the weak turbulence approach may be used. It is based, as a rule, on the random phase approximation, thus reminding the incoherent light. The most typical window of parameters they use to determine the limits of its applicability, may be determined by the 'degree of turbulence' $W / n T$ where $W$ is the energy density of oscillations and $n T$ the thermal energy density. For example, for the Langmuir waves, the most typical plasma mode, they use the following chain of inequalities:

$$
1 \gg \frac{W}{n T} \gg \frac{1}{N_{\mathrm{D}}}
$$

Here $N_{\mathrm{D}} \gg 1$ is the number of particles in a sphere of the radius equal to the Debye length $r_{\mathrm{D}}$. Only great $N_{\mathrm{D}}$ typical of the hot and/or rarefied plasmas allow the predomination of the collective effects. One of the important features of the weakly turbulent regime is the 'inverted' energetic flux over scales, compared to the strong, say, Kolmogorov turbulence (see Fig. 1(a,b)). Indeed, the main process of nonlinear dissipation within the frames of weak (perturbational) turbulence is the stimulated 

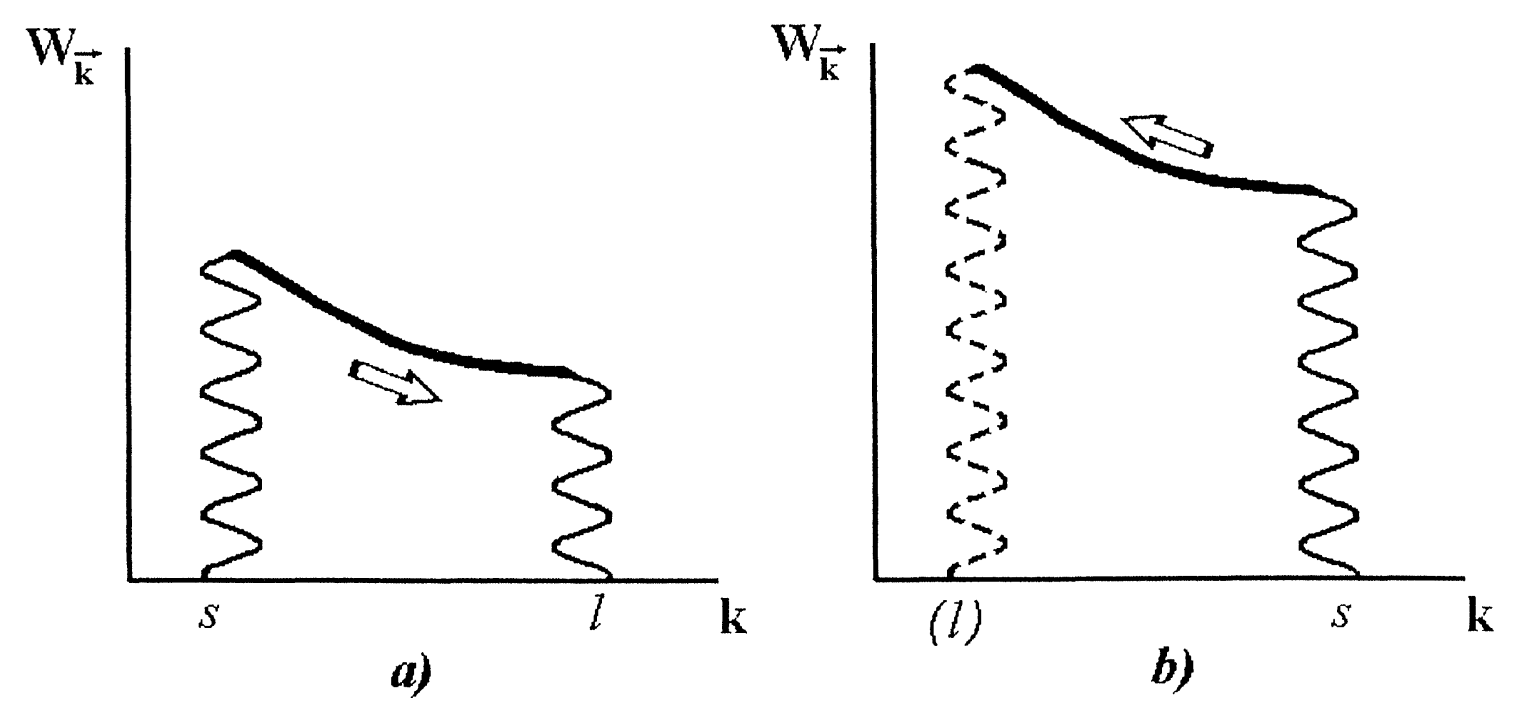

FIGURE 1 Energetic flux over scales: (a) strong (hydrodynamic) turbulence, (b) weak (perturbational) turbulence.

scattering of waves by particles in which the number of quanta remains to be invariant, thus, growing entropy results in reducing frequency of waves. In the case of normal dispersion (typical of quasisteady plasmas) it is equivalent to the dynamics presented in Fig. 1.

Meanwhile, only in very few cases of the strong turbulence the left inequality becomes violated, so that $W \sim n T$. For example, in hydrodynamics, it would mean the oscillating velocity being of the order of the acoustic velocity. Much more typical of strongly turbulent regimes are the effects of coherence, phase correlation and, after all, the nonlinear structures. Thus, the question arises: can such a regime be turbulent or not? The matter is that superposition principle breaks in essentially nonlinear media (cf., e.g., steady magnets), thus, at first sight, turbulent behavior becomes impossible.

This is the general problem, however, the physical community started studying strong Langmuir turbulence following, first of all, the problems of laser fusion. The typical mechanism of the energetic input into the plasma corona of a laser target was collective one even for $\mathrm{Nd}$ lasers $(\lambda=1.06 \mu)$ and moreover for $\mathrm{CO}_{2}$ lasers $(\lambda=10.6 \mu)$. The collective absorption was being based on the parametric instabilities, as a result, the main fraction of energy put in turned out to transfer just to the Langmuir waves. The latters, within the frames of the weakly turbulent regime. would lose their energy in average, little by little. Let us follow the dispersive relation of the Langmuir waves,

$$
\omega=\omega_{\text {pe }}\left[1+\frac{3}{2}\left(k r_{\mathrm{D}}\right)^{2}\right], \quad k r_{\mathrm{D}} \ll 1
$$

with $\omega_{\text {pe }} \equiv\left(4 \pi n e^{2} / m\right)^{1 / 2}$ being the electron plasma frequency and $r_{\mathrm{D}}$ Debye radius. One can readily see that the overwhelming fraction of the oscillatory energy remains frozen in the long-wave plasma waves with $\omega \simeq \omega_{\text {pe }}$. There is the only mechanism of damping of these waves, and not so efficient one, that is collisional damping. Let us note once more that such a scenario turns out to be opposite to that of the conventional Kolmogorov turbulence, in which the source in the $k$-space corresponds to the longer scales while the leakage of the energy of oscillations is usually located in small space scales.

At first sight, that means the resulting accumulation of the wave energy in a plasma corona, that is so-called condensation of plasmons. In fact, 
nonlinear effects join the game, first of all, modifying the dispersion law:

$$
\omega=\omega_{\mathrm{pe}}\left[1+\frac{3}{2}\left(k r_{\mathrm{D}}\right)^{2}-\beta \frac{W}{n T}\right]
$$

where $\beta \sim 1$ and $W$ is the energy density of the plasma waves. It is interesting to compare (1) with the relativistic form of the energy of a nonrelativistic particle:

$$
\mathcal{E}=m c^{2}+\frac{p^{2}}{2 m}+U(\mathbf{r})
$$

Weak turbulence is nothing but the perturbation theory based on the zero level representation of the oscillating field as an ensemble of the noninteracting waves (quasi-free plasmons). Let us note that, usual particles in (2) remain almost free and their energy may be estimated as $p^{2} / 2 m$ only if $U \ll$ $p^{2} / 2 m$. It is no use to compare their potential energy with $m c^{2}$. Respectively, the validity of the weak turbulence theory for Langmuir plasmons should be estimated as

$$
W \ll n T\left(k r_{\mathrm{D}}\right)^{2} .
$$

If this inequality becomes violated, intermode coupling turns out to be strong even on the level of zero order approximation. Although such a turbulence is not strong in the sense of separation of oscillating and random particle motion, the quasiparticles have to be built on the base of the renormalized theory. Thus, in fact, the parameter of expansion while constructing the weak turbulence theory has to be not $W / n T$ but $W /\left[n T\left(k r_{\mathrm{D}}\right)^{2}\right]$. First, it was established by Vedenov and Rudakov, 1965 (see, e.g., [2,3]). In particular, it was shown that, as a result of violation of (3) inequality, the specific modulational instability had to start resulting in the localization of plasmons in some clots or drops. In other words, instead of a homogeneous weak turbulence, nonlinear structures would arise.

\section{BASIC EQUATIONS. LANGMUIR SOLITON}

What does occur after weakly turbulent treatment becomes broken? It looks not incredible that we can proceed something like the quasiparticle formalism. However, the quasiparticles themselves have to be chosen of the new form and with some new properties. Plane wave (or another linear approach) does not fit more. Of course, Fourier expansion may be used in any case. But in general we have to study plane wave ensemble including fast varying phases, not only spectral intensities. To escape the violation of the main basic property of the stationarity of numbers of particles in each initial and final state we should search for a new kind of quasiparticles. Let us remind that modulational instability results in the localization of the oscillatory field, in other words, chaotic turbulence tends to the transformation into the nonlinear structures. Thus, it seems reasonable to start from the nonlinear equations in the $x$-space without using the Fourier transform. To separate oscillating and slow evolution of all the physical parameters, the following substitution is useful to use:

$$
\overrightarrow{\mathcal{E}}(\mathbf{r}, t)=\frac{1}{2}\left[\mathbf{E}(\mathbf{r}, t) \exp \left(-\mathrm{i} \omega_{\mathrm{pe}} t\right)+\text { c.c. }\right]
$$

with $\overrightarrow{\mathcal{E}}(\mathbf{r}, t)$ being the complex amplitude and $t$ the 'slow' time, i.e., $\partial / \partial t \ll \omega_{\text {pe }}$. The nonlinear dynamics turns out to obey Zakharov equations [4]:

$$
\begin{gathered}
\operatorname{div}\left[2 \mathrm{i} \frac{\partial \mathbf{E}}{\partial t}+3 \omega_{\mathrm{pe}} r_{\mathrm{D}}^{2} \nabla \operatorname{div} \mathbf{E}-\omega_{\mathrm{pe}} \frac{\delta n}{n} \mathbf{E}\right]=0, \\
\left(\frac{\partial^{2}}{\partial t^{2}}-c_{\mathrm{s}}^{2} \nabla^{2}\right) \delta n=\nabla^{2} \frac{|E|^{2}}{16 \pi M_{\mathrm{i}}},
\end{gathered}
$$

where $\delta n$ is the density perturbation, $c_{\mathrm{s}}=\sqrt{T_{\mathrm{e}} / M_{\mathrm{i}}}$ ion acoustic velocity, all other terms are conventional. We keep the operator 'div' in the LHS of (5) since two first terms are the potential vectors but the third. To keep the correct space symmetry we have to keep 'div' but only in the 1-D case. In the linear approximation, the systems (5) and (6) become 
splitted resulting in the independed acoustic motion with immaterial HF pressure in the RHS of (6), and linear dispersion of Langmuir waves, in accordance with (1).

For simplicity, only 1-D case will be considered in this paper. It is useful to note that 3-D dynamics is essentially different (see [2,4]).

Let us assume a very slow motion when both electrons and ions are permitted to be described by the Boltzmann distribution:

$$
\begin{gathered}
n_{\mathrm{e}}=n_{0} \exp \left(\frac{e \Phi}{T}-\frac{P_{\mathrm{HF}}}{n_{0} T}\right)=n_{\mathrm{i}}=n_{0} \exp \left(-\frac{e \Phi}{T}\right), \\
P_{\mathrm{HF}} \simeq \frac{|E|^{2}}{16 \pi} \ll n T,
\end{gathered}
$$

where $\Phi$ is the potential of the charge separation. Thus, HF pressure repells the electrons, they, in turn, pull out the ions, as a result, the self-consistent density well becomes formed in which the oscillating field is 'locked'. It is just the result of the modulational instability. Then one can exclude $\Phi$ :

$$
e \Phi=P_{\mathrm{HF}} / 2 n_{0} \Rightarrow \delta n_{\mathrm{i}} \simeq-n_{0} \frac{|E|^{2}}{16 \pi n_{0} T} .
$$

Together with (5), it results immediately in the nonlinear Schrödinger equation (NSE),

$$
\mathrm{i} \frac{\partial E}{\partial t}+\frac{3}{2} \omega_{\mathrm{pe}} r_{\mathrm{D}}^{2} \frac{\partial^{2} E}{\partial x^{2}}+\omega_{\mathrm{pe}} \frac{|E|^{2}}{32 \pi n M_{\mathrm{i}}} E=0 .
$$

This equation has been studied well enough. It is known that it has the infinite set of integrals of motion. In particular, it means that no turbulence is permitted to exist within the framework of (7) but only the entirely determinate nonlinear dynamics. We have, not to forget, however, that (7) is not more than the quasi-steady or essentially subsonic model of the Langmuir dynamics. It is interesting to note that essentially subsonic limit of Eqs. (5) and (6) is the same NSE. Meanwhile, in one point this system is opposite to (7) since it includes hydrodynamic description (6) of the background (i.e., ions) which is opposite limit with respect to the Boltzmann distribution. Respectively, the same effect of the field localization follows from (5) and (6) but

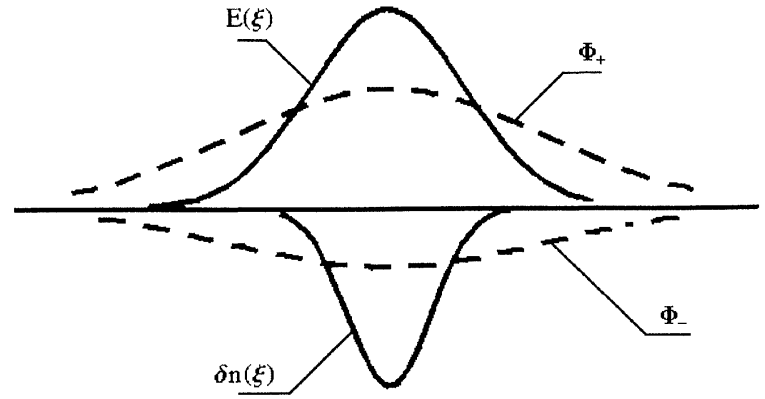

FIGURE 2 Langmuir soliton.

conditioned by the different mechanism. To wit, the ion well in this case is the consequence not of the potential hill but of the potential well through which ions are flowing faster and $\delta n<0$ follows from the continuity of the ion flux. Both cases are presented in Fig. 2. Let us turn to the exact solution describing this effect of localization.

Fundamental object in the strong Langmuir turbulence is called Langmuir soliton (Rudakov, 1972, see, e.g., [2,3]). It can be obtained analytically starting from Eqs. (5) and (6). We will search for this solution in a form of travelling wave:

$$
\begin{aligned}
E(x, t) & =E\left(x-v_{\mathrm{s}} t\right) \exp [\mathrm{i}(k x-\delta \omega t)], \\
\delta \omega & \equiv \omega-\omega_{\mathrm{pe}}, \quad E_{x \rightarrow \pm \infty} \rightarrow 0 .
\end{aligned}
$$

Here $v_{\mathrm{S}}$ is the soliton velocity (not acoustic velocity $c_{\mathrm{s}}$ ), the frequency shift $\delta \omega$ includes both dispersive and nonlinear effects. Space modulation $(k)$ is inevitable if $v_{\mathrm{s}} \neq 0$, as it will be seen below.

It is convenient, for simplicity, to start with substitution of (8) in NSE which turns out to be splitted in two:

$$
\begin{gathered}
\frac{3}{2} \omega_{\mathrm{pe}} r_{\mathrm{D}}^{2} E_{\xi \xi}+\left(\delta \omega-\frac{3}{2} \omega_{\mathrm{pe}} k^{2} r_{\mathrm{D}}^{2}\right) E \\
+\omega_{\mathrm{pe}} \frac{|E|^{2}}{32 \pi M_{\mathrm{i}}} E=0 . \\
-v_{\mathrm{s}} E_{\xi}+3 k \omega_{\mathrm{pe}} r_{\mathrm{D}}^{2} E_{\xi}=0 .
\end{gathered}
$$

where $\xi=x-v_{\mathrm{s}} t$. (10) immediately results in

$$
v_{\mathrm{s}}=3 k \omega_{\mathrm{pe}} r_{\mathrm{D}}^{2} \equiv \frac{\partial \omega^{l}(k)}{\partial k} .
$$


and that is just the argument for the space modulation. Indeed, the nonlinear wave velocity turns out to be equal to the group velocity of the Langmuir waves, hence, $v_{\mathrm{s}} \neq 0 \Rightarrow k \neq 0$. The difference within the brackets in the LHS of (9) is the nonlinear shift of the frequency while the total shift may be presented in the form

$$
\partial \omega=\frac{3}{2} r_{\mathrm{D}}^{2}\left(k^{2}-k_{0}^{2}\right),
$$

where $k_{0}$ will be found while solving (9), together with $E(\xi)$ dependence:

$$
E=\frac{E_{0}}{\cosh k_{0} \xi}, \quad k_{0}=\frac{e E_{0}}{\sqrt{24} T} .
$$

This is the only nonlinear formation stable with respect to the modulational instability, and consequently, the final result of this instability. Unlike KDV solitons, it includes HF modulation and depends on two free parameters, viz., $E_{0}$ and $v_{\mathrm{s}}$ (or $k$ ). If, instead of NSE, one solves the full system of Zakharov equations (5) and (6), exact solutions will be slightly different from (12) due to the 'relativistic' effects:

$$
\begin{gathered}
E=\frac{E_{0}}{\cosh k_{0} \xi}, \quad \xi=x-v_{\mathrm{s}} t, \delta \omega=\frac{3}{2} r_{\mathrm{D}}^{2}\left(k^{2}-k_{0}^{2}\right), \\
k_{0}=\frac{e E_{0}}{\sqrt{24}\left(1-\nu_{\mathrm{s}}^{2} / c_{\mathrm{s}}^{2}\right) T} .
\end{gathered}
$$

It is interesting to consider the Fourier spectrum of the Langmuir soliton. Funnily enough, it turns out to be presented by the same function $\cosh ^{-1}$ :

$$
\begin{aligned}
E(x, t)= & \operatorname{Re} \int_{-\infty}^{+\infty} \mathrm{d} k^{\prime} \frac{E_{0}}{2 k_{0} \cosh \left(\pi k^{\prime} / 2 k_{0}\right)} \\
& \times \exp \left[\mathrm{i}\left(k^{\prime}+k\right) x-\mathrm{i}\left(\omega+k^{\prime} v_{\mathrm{s}}\right) t\right] \\
\equiv & \operatorname{Re} \int_{-\infty}^{+\infty} \mathrm{d} q E_{q} \exp \left[\mathrm{i}\left(q x-\Omega_{q} t\right)\right], \\
E_{q}= & \sqrt{6} \sqrt{1-v_{\mathrm{s}}^{2} / c_{\mathrm{s}}^{2}} \frac{T}{e \cosh \left[\pi(q-k) / 2 k_{0}\right]}, \\
\Omega_{q}= & \omega_{\mathrm{pe}}+\delta \omega+(q-k) v_{\mathrm{s}} .
\end{aligned}
$$

Fourier spectra of both standing and travelling Langmuir solitons are drawn in Fig. 3. At least two

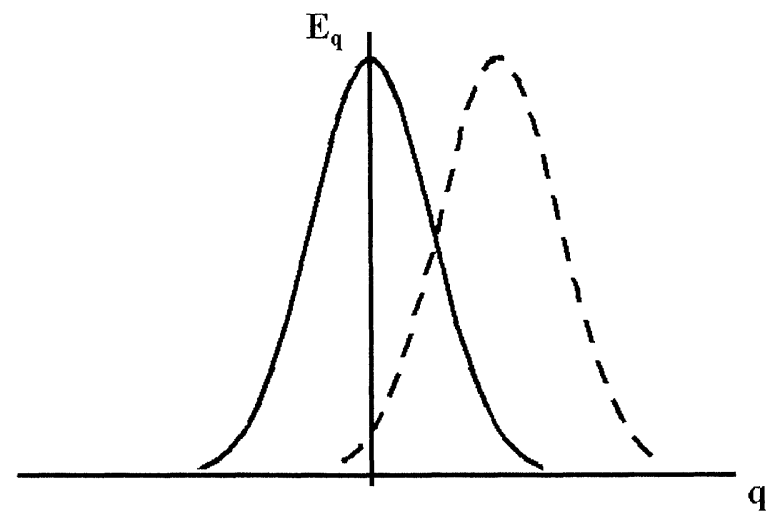

FIGURE 3 Fourier spectra of both immovable (solid line) and travelling (dotted line) Langmuir solitons.

of their interesting properties should be pointed out:

(1) The amplitude of the spectral distribution in the $k$-space does not depend of $E_{0}$ but only the spectral width. This is the evident consequence of the fundamental relation $k_{0} \propto E_{0}$.

(2) $\left(\Omega_{q}-\Omega_{q}^{\prime}\right) /\left(q-q^{\prime}\right) \equiv v_{\mathrm{s}}$. This is a manifestation of the nature of the fundamental nonlinear processes involved into the problem:

$$
l \rightarrow l+s, \quad l+l \rightarrow(s) \rightarrow l+l .
$$

Thus, the low-frequency component of the Langmuir soliton (see $\delta n$ in Fig. 3) is the commom beat of all the HF harmonics.

\section{SOLITON MODEL OF THE STRONG LANGMUIR TURBULENCE}

First of all, let us emphasize that, unlike NSE, not the infinite number of integrals of motion may be introduced for Zakharov equations (5) and (6) but only three, to wit, the number of quanta:

$$
I_{0}=\frac{1}{4 \pi \omega_{\mathrm{pe}}} \int_{-\infty}^{+\infty}|E|^{2} \mathrm{~d} x
$$

the integral of momentum $I_{1}$, and the dispersive fraction of the oscillatory energy (like $\mathcal{E}-m c^{2}$ for 
the particles):

$$
I_{2}=\mathcal{E}-\omega_{\mathrm{pe}} I_{0} .
$$

As a result, Eqs. (5) and (6) are not completely integrable and the turbulent behavior is allowed within the frames of the model based on (5) and (6).

Equations (5) and (6) can describe both modulational instability and essentially nonlinear dynamics. As the modulational instability is conditioned by the level of turbulence high enough, $W / n T \geq$ $\left(k r_{\mathrm{D}}\right)^{2}$, one could expect its final result to be, at least, $W / n T \simeq\left(k_{0} r_{\mathrm{D}}\right)^{2}$ where $k_{0}$ is an effective wave number corresponding to some typical scale of localization, $L \sim k_{0}^{-1}$. If $W \rightarrow n T$ due to the pumping (laser or particle beam or something else), effective damping process joins the game, that is Landau damping since $k_{0} r_{\mathrm{D}} \rightarrow 1$ providing the dissipation. Thus, the flux of energy in the $k$-space becomes inverted with respect to the weak turbulence, and all the turbulent scenario acquires the typical features of the Kolmogorov-like turbulence.

As it has been noticed above, to represent any turbulent behavior, the superposition principle has to be provided by the model being used. From this point, Langmuir solitons are looking rather attractive to play the role of new quasiparticles since these coalescenes are restricted in space with exponential accuracy, hence, the superposition principle can be satisfied with the same accuracy. Besides, except of the amplitude, each soliton has one more free parameter, i.e., velocity, that allows to form real chaotic behavior of the resulting field. In addition, solitons of different amplitudes, have also different width, thus, one of them seems to be something like quasiclassical well for another, as a result, these solitons may pass free one through another, like KDV solitons do. After all, unlike any other wave formation, soliton is stable with respect to the modulational instability.

Following Eqs. (12) and (13), to wit, $k_{0}\left(E_{0}\right)$ dependence, one readily can see that self consistent relation $W / n T \simeq\left(k_{0} r_{\mathrm{D}}\right)^{2}$ is true for any particular soliton. Taken as averaged in space, $\langle W\rangle / n T$ may even essentially less than $\left(\left\langle k_{0}\right\rangle r_{\mathrm{D}}\right)^{2}$, thus, to differ weak turbulence from the strong one, it is useful to follow the direction of the energy flux in the $k$-space.

The fundamental assumption was made in [1] that this flux in the strongly turbulent regime was provided by the soliton fusion 'two in one' in which only solitons with close amplitudes could take part. Indeed, integral of motion $I_{0}$ allows this process (and return process as well since $I_{0} \propto E_{0}$ ). In turn, integral of motion $I_{2}$ allows the process of fusion but forbids the process of decay, 'one in two'. As for the close amplitudes, this assumption was made to escape the multi-soliton collapse, based on the idea of quasiclassical approach mentioned above. Both assumptions were confirmed later in the 'computer experiment' carried out by Degtyarev et al. [5]. Its results added only one but essential circumstance to the model concerning the important role of the acoustic waves (background noises) in the dynamics of multi-soliton systems. The qualitative picture of the coalescence 'two in one' following from these simulations is presented in Fig. 4.

Let us put $W$ to be the average density of the turbulent state and $L$ - the length of the 1-D turbulent plasma system. Then let us introduce the set of the fundamental states in assumption that in each state the turbulent energy is distributed between $N$ identical solitons so that $N$ is the parameter of state. Their amplitude may be determined by using the equality

$$
W L=N \mathcal{E}_{0}(N)=\frac{\sqrt{6}}{2 \pi e} E_{0}(N) N T .
$$

The maximal number of solitons in a state is restricted by the condition of close packing $N_{\max } \simeq$ $k_{0} L$, which yields

$$
N_{\max } \simeq \frac{1}{2 \sqrt{6}}\left(\frac{W}{n T}\right)^{1 / 2} \frac{L}{r_{\mathrm{D}}} .
$$

The minimal number of solitons is conditioned by the Debye scale, or, if there exists suprathermal 'tail' of electrons, by some $k_{0 \max } \leq r_{\mathrm{D}}^{-1}$ which determines the cut off the turbulent spectrum:

$$
N_{\min } \simeq \frac{1}{24} \frac{W}{n T} \frac{L}{r_{\mathrm{D}}}\left(k_{0 \max } r_{\mathrm{D}}\right)^{-1} .
$$



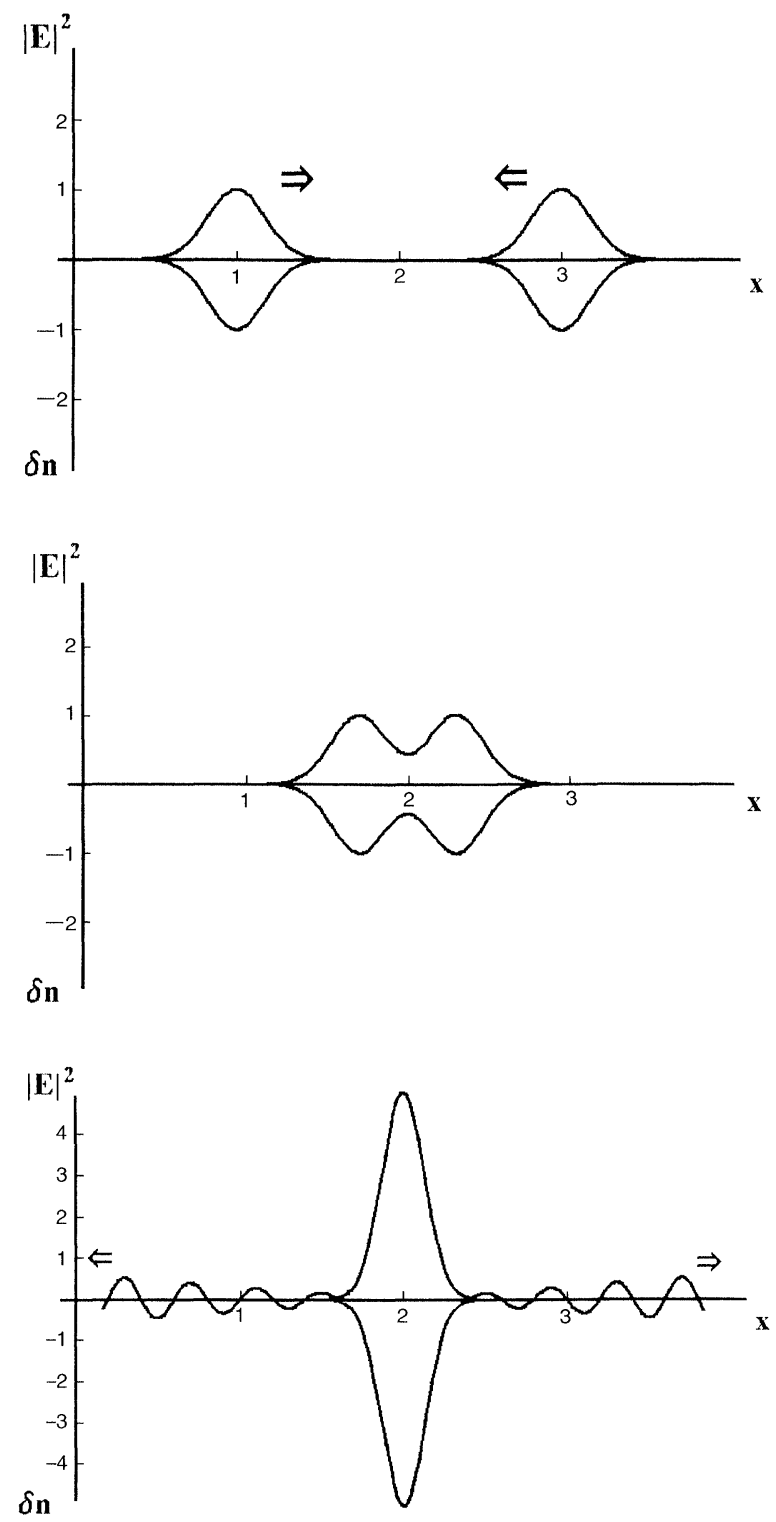

FIGURE 4 Three stages of the process of fusion of two Langmuir solitons.

In a result, the turbulent state may be presented in the form of expansion over fundamental states. Let us define $P(N)$ as the probability of $(N)$ state:

$$
P(N)=\frac{\Delta n(N)}{N},
$$

where $\Delta n(N)$ is the number of solitons of the amplitude $E_{0}(N)$ in the real state. Hence, $P(N)$ is also the fraction of the total energy provided by these solitons as $\mathcal{E} \propto E_{0}$ :

$$
\Delta \mathcal{E}(N)=\mathcal{E}_{0}(N) P(N) .
$$

As a rule, in the computer simulations they follow the energetic spectrum in the $k$-space (the same had been studied in the kolmogorov model). Well, let us calculate the spectral intensity $W_{k}$ depending on the $P(N)$ distribution. For this purpose the expansion (14) will be used. In the turbulent state, all the solitons have to be placed randomly, with random phases. Thus, the squares of their harmonic amplitudes are allowed to be summarized:

$$
W_{k}=\frac{12 T^{2}}{L e^{2}} \int_{N(\min )}^{N(\max )} \mathrm{d} N N P(N) \cosh ^{-2}\left(\frac{3 T^{2} N k}{e^{2} W L}\right) .
$$

Roughly, for simplicity, $\cosh ^{-2}(x)$ may be estimated by the step function $\Theta(1-|x|)$ which cuts off the integration at

$$
N_{0} \simeq \frac{1}{3}\left(\frac{e}{T}\right)^{2} \frac{W L}{k}<N_{\max } .
$$

Thus, the final result may be presented as

$$
W_{k}=\frac{12 T^{2}}{L e^{2}} \int_{N(\min )}^{N(0)} \mathrm{d} N N P(N) .
$$

In many cases, spectra of strong Langmuir turbulence obtained in simulations may be well approximated by the function

$$
W_{k} \propto k^{-2},
$$

which in our representation corresponds to

$$
P(N)=\text { const, }
$$

thus being an analog of the Rayleigh-Jeans distribution. (In a weakly turbulent regime, i.e., in the Fourier representation, this distribution degenerates to $W_{k}=$ const $\equiv 2 T / \pi$.)

Our representation may be without difficulties translated into more usual treatment, operating not 
with the probability $P(N)$ but with the averaged number of solitons of the given amplitude per unit length, i.e., with the soliton distribution function:

$$
F\left(E_{0}\right)=P(N) N \frac{\mathrm{d} N}{\mathrm{~d} E_{0}} .
$$

Particularly, 'Rayleigh-Jeans distribution' given by (24) becomes transformed into the following:

$$
F\left(E_{0}\right) \propto E_{0}^{-3} .
$$

\section{NUMERICAL SIMULATIONS}

Basic assumptions of [1] were confirmed in [5]. Besides, some additional features of this 'relay race' turbulent model were revealed. In a whole, only rather few fundamental problems of such a nonlinear physics may be solved analytically. It can be very important in the subsequent construction of theoretical 'images' of real dynamics, however, most results upto-date have been obtained by the simulations. In the paper [5] real turbulence had been studied, based on the energetic flux over scales. It was shown that not only radiation of the ion-acoustic waves was typical of this turbulence, not less important was the soliton fission by the acoustic quanta radiated by the fusion of more intensive solitons. The real spectra of strong Langmuir turbulence were calculated, in fact very similar to $(24,26)$.

In many later papers not the turbulent behavior was being studied but a kind of thermodynamics which was thermodynamics of waves only, without their thermodynamical equilibrium with particles. In principle, it is different problem, however, it can be easier posed and more carefully modelled in simulations. Resulting tendency may be expanded onto the turbulent regime since the fundamental interactions are the same in both cases. The most interesting are the papers [6-8] which demonstrate 'dew' effect in the strong Langmuir turbulence. As the drops of water interact in a closed volume via the vapor, solitons with the HF filling ('solitons of envelope') interact via the weakly turbulent spectrum. The well known result of thermodynamics is either the total evaporation of all the drops or their condensation in the only drop being in dynamic equilibrium with the vapor. Also solitons either become converted into the weakly turbulent ensemble or tend to the formation of the only soliton of higher amplitude being in dynamic equilibrium with the weak turbulence. This result was obtained numerically in [6-8], it implicitly cofirms the basic concept of the soliton model of turbulence [1].

\section{CONCLUSION}

Thus, it has been shown that strong turbulence of the plasma waves combines two basic properties of the nonlinear dynamics, viz., turbulent behavior and nonlinear structures. The latters can be modelled in one dimension by specific two-parametric solitons with HF modulation. Perhaps, this model can be expanded, in principle, onto some other nonlinear dynamics based on the interaction of individual objects with some individual properties resulting in the chaotic behavior that, in turn, results in some macroscopic dynamics. This dynamics includes the irreversible processes and may be contemplated, in a whole, as the complicated dynamical dissipative structure.

\section{References}

[1] Kingsep, A.S., Rudakov, L.I. and Sudan, R.N.: Phys. Rev. Letters, 31, 1482 (1973).

[2] Kingsep, A.S.: in: Itogi Nauki i Tekhniki (in Russian, Progress in Science and Technology Series on Plasma Physics) V.D. Shafranov. Ed., Moscow. VINITI, Vol. 4, pp. 48-113 (1984).

[3] Kingsep, A.S.: Introduction to Nonlinear Plasma Physics (in Russian), Moscow, MIPT (1996).

[4] Zakharov, V.E.: Sov. Physics JETP, 62, 1745 (1972).

[5] Degtyarev, L.M., Makhan'kov, V.G. and Rudakov, L.I.: Sov. Physics JETP, 67, 533 (1974).

[6] Krylov, V.F. and Yankov, V.V.: Models of Strong Turbulence, Technical Report 3542/6, Kurchatov Institute, Moscow (1982).

[7] Zakharov, V.E., Pushkarev, A.N., Shvets, V.F. and Yankov, V.V.: JETP Lett., 48(2), 83 (1988).

[8] Dyachenko, A.I., Zakharov, V.E., Pushkarev, A.N., Shvets, V.F. and Yankov, V.V.: Sov. Phys. JETP, 96, 2026 (1989). 


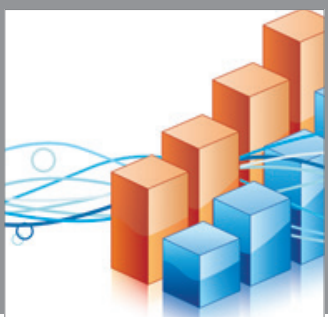

Advances in

Operations Research

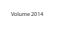

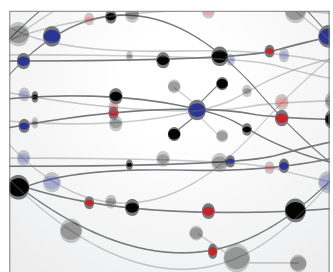

\section{The Scientific} World Journal
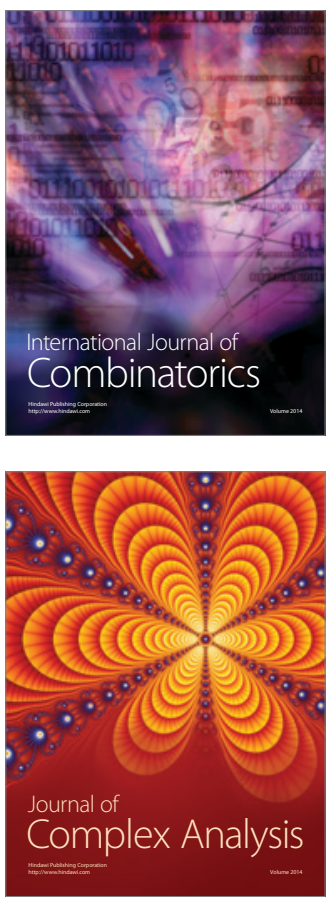

International Journal of

Mathematics and

Mathematical

Sciences
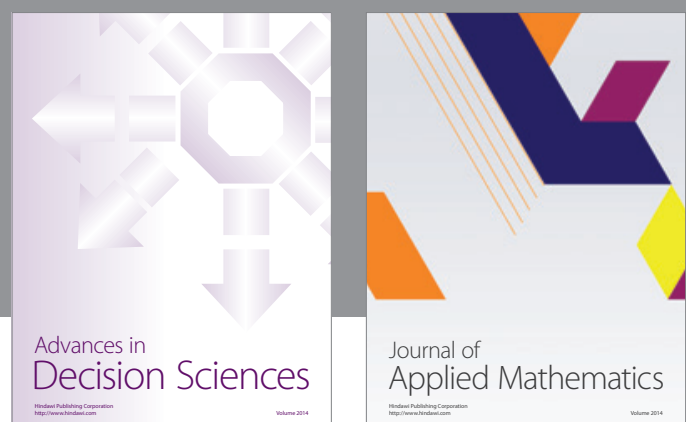

Journal of

Applied Mathematics
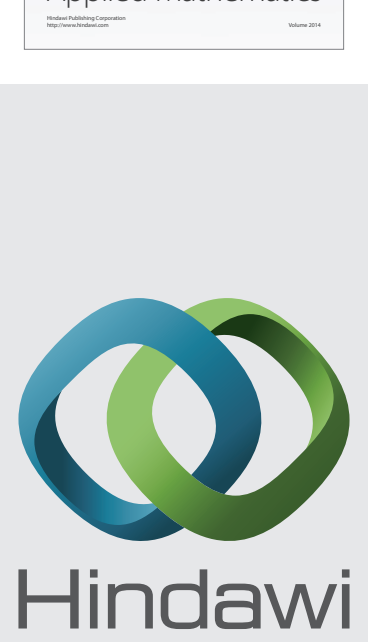

Submit your manuscripts at http://www.hindawi.com
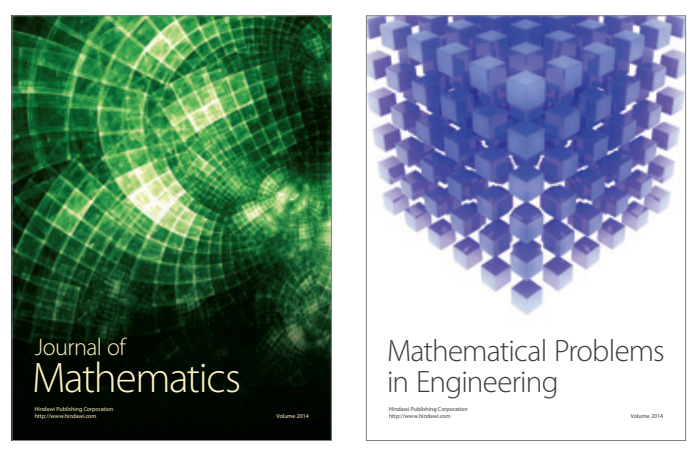

Mathematical Problems in Engineering
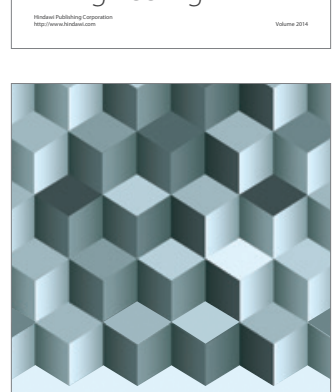

Journal of

Function Spaces
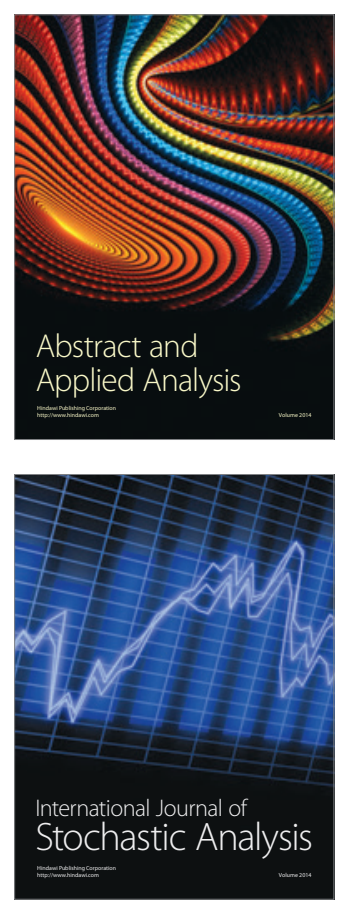

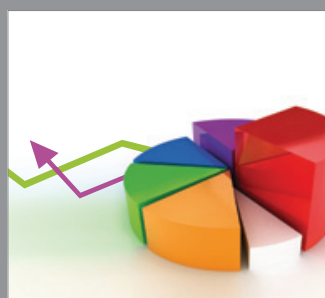

ournal of

Probability and Statistics

Promensencen
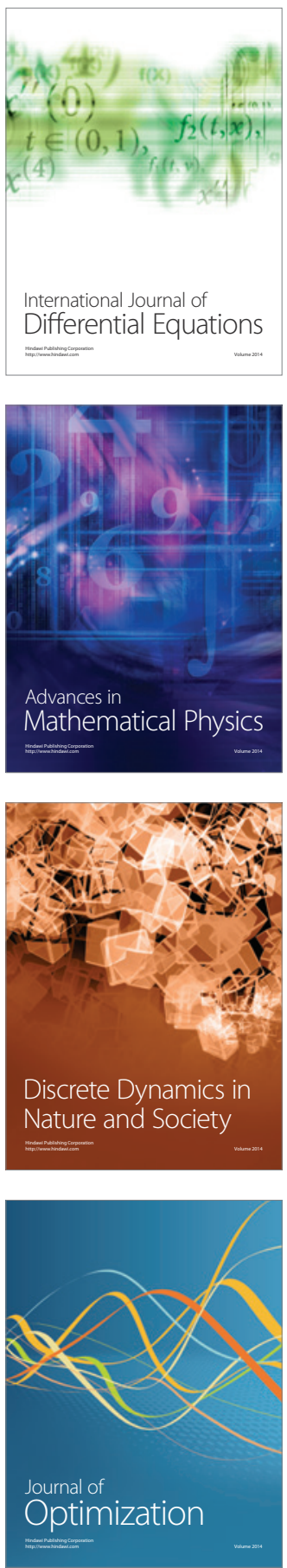